\title{
Electronic Structures of Silicene Doped with Galium: First Principle study
}

\author{
Mauludi Ariesto Pamungkas ${ }^{1, a}$, Wafa Maftuhin ${ }^{2, b}$ \\ 1,2 Jurusan Fisika (Physics Department), Universitas Brawijaya, Indonesia
}

\begin{abstract}
Following the success of graphene which possesses unique and superior properties, 2D material other than graphene become centre of interest of material scientists. Silicene, which has the same crystal structure as graphene but consist of silicon atoms rather than carbon become intriguing material due to domination of silicon as main material of electronic component. It is common to enhance electronic properties of semiconductor by adding dopant atoms. The electronic properties of Silicene doped with Gallium are investigated using first principle calculation based on density functional theory (DFT).Ga doping changes character of silicene from semimetal to conductor except silicene with Ga doping on S-site ( $\mathrm{Ga}$ atom substitutes one $\mathrm{Si}$ atom) which lead to semiconductor.
\end{abstract}

\section{Introduction}

Today, graphene has attracted the most attention of material researchers not only because it is the first 2D material successfully synthesized but also because of its peculiar behaviors and superior properties[1]. Its successful synthesis and potential applications in wide areashave stimulated many researchers to study possibility of elements other than carbon to be formed ingraphene structure. Considering domination of silicon in electronic industries owing to its abundant existence in the earth as well as excellent interface with its oxide, investigation of silicene, silicon material with graphene crystal structure (two dimensional honey comb), is paramount important. Theoretical prediction of the existence of silicene had been started even before invention of graphene[2]. Despite possibility of silicene to exist had ever been questioned due to natural $\mathrm{sp}^{3}$ hybridization of siliconsilicene has been successfully synthesized on a Ag (111) substrate[3].Recently, silicene has been successfully implemented as a channel layer of filed effect transistor[4]. Other important research findings from recent theoretical studies have shown that electronic band structure of silicone is similar to that of graphene. However,silicine has larger band gap at Dirac point.In addition, due to its low buckle, band gap of silicene can be controlled by electric fields[5,6]. Like graphene, Siliceneposses massless Dirac fermion whoseelectron velocity is only hundredths of light velocity[7]. Recent first principle study reported existence of quantum hall effect in silicene as it was found in graphene[8]. Considering above mentioned both similarities and differences between grapehene and silicine, it is then justified to expect similarities and differences in other properties.

Beside properties of prisitinegraphene, graphene doped with other elements, particularly from third and fifth column of periodic table, have been intensively investigated. Doping on graphene influences its electronic structure especially tune its band gap. Therefore it is intriguing to investigate influences of doping on electronic structure of silicene. This work is aimed to investigate influence of Gallium doping onthe electronic structure of silicene.

\section{Computational Methods}

The calculations are performed using Density functional Theory implemented in ABINIT code. The electron-ionic core interaction is represented by norm conserving pseudopotential. Perdew-Burke-Ernzerhof (PBE) formulation of the GGA is chosen to treat electron exchange correlation. Plane wave basis set with cutoff energy of 20 hartree $(560 \mathrm{eV})$ is used.Special $12 \times 12 \times 1 k$ point grid was generated using Monkhorst-Pack scheme.

We studied Silicene with armchair structure and with size of $2 \times 2$ cell. Vacuum layer with width of 15 angstrom is implemented to eliminate interaction.One Gallium adatomis placed on several most possible positions. The system is then relaxedwith BroydenFletcher-Goldfarb-Shanno (BFGS) algorithmto lead it into ground state with forces smaller than $0.005 \mathrm{eV} / \mathrm{A}$.

\section{Results and Discussion}

At first, we calculated ground state structure and electronic properties of baregraphene and silicene to confirm our calculation parameter. The equilibrium lattice constant of bare graphene and silicene are $2.46 \mathrm{~A}$ and $3.90 \mathrm{~A}$ respectively which are close to the values reported by previous studies[9],[10],[11], [12].

When Silicine is doped with Galium atom in the B-site,Galium atom whose atomic radius is larger than that of silicon atom is placed in the middle of honey

\footnotetext{
$\bar{a}$ m_ariesto@ub.ac.id, ${ }^{b}$ wafamaftukhin@gmail.com
} 
comb structure surrounded by silicon atoms. After relaxation, the closest silicon atom is pushed out to find new stable position so that $\mathrm{Si}-\mathrm{Si}$ bonding is broken. Closest distance between $\mathrm{Ga}$ atom and silicon atom after they reach stable position is within 2.87-2.99 Angstrom. For doping of $\mathrm{Ga}$ atom on $\mathrm{T}$-site, namely putted above one $\mathrm{Si}$ atom, after the system is relaxed, the closest silicon atom is also repelled away and leads to breaking of $\mathrm{Si}-\mathrm{Si}$ bond. The closest $\mathrm{Ga}-\mathrm{Si}$ distance is 2,63 Angstrom. While, for doping at $\mathrm{H}$-site, there is no position of atoms change significantly. In this case, Ga bonds to $\mathrm{Si}$ atom with bond length of 2.32 Angstrom.Differ from other Ga position, doping of $\mathrm{Ga}$ atom on $\mathrm{S}$-site, $\mathrm{Ga}$ atom replaces one $\mathrm{Si}$ atom position.

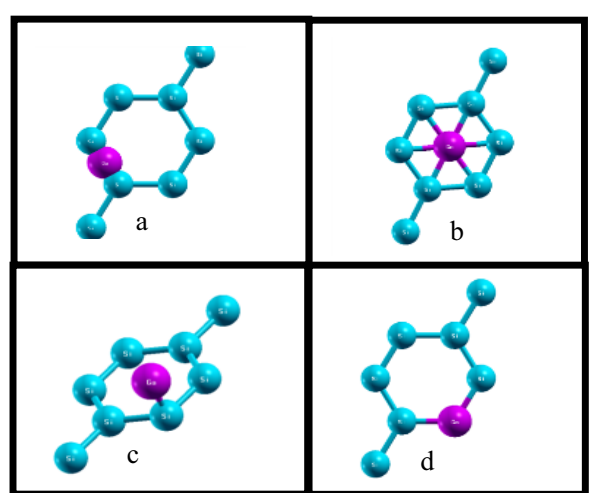

Figure 1. Position of $\mathrm{Ga}$ atom on silicene, a. Brige (B-site), b. Hollow (H-site), c. Top (T-site), and d.Substitute (S-site)

Table 1 Structure and formation energy of silicene doped with $\mathrm{Ga}$ atom.

\begin{tabular}{|l|l|l|l|c|}
\hline $\begin{array}{l}\text { Position of } \\
\text { Ga atom } \\
\text { doping }\end{array}$ & $\begin{array}{l}\text { Si-Si } \\
\text { (Angstro } \\
\text { m) }\end{array}$ & $\begin{array}{l}\text { Si-Ga } \\
\text { (Angstro } \\
\text { m) }\end{array}$ & $\begin{array}{l}\text { Ef } \\
\text { (eV) }\end{array}$ & Eg \\
\hline GA top site & 2.29 & 2.94 & - & - \\
\hline $\begin{array}{l}\text { GA bridge } \\
\text { site }\end{array}$ & $2.12-2.18$ & $2.87-2.99$ & - & - \\
\hline $\begin{array}{l}\text { GA Hollow } \\
\text { site }\end{array}$ & 2.3175 & 2.32 & - & - \\
\hline $\begin{array}{l}\text { Substitution } \\
\text { GA }\end{array}$ & $2.22-2.26$ & 2.25 & $\begin{array}{l}- \\
14,81\end{array}$ & 0,275 \\
\hline
\end{tabular}

Table 1 shows that silicene with doping of Ga atom at $\mathrm{S}$-site is the most stable state. Formation energy is calculated based on this equation :

$$
\mathrm{E}_{\text {formation }}=\left(\mathrm{E}_{\text {total }}-\mathrm{E}_{\text {silicene }}-\mathrm{E}_{\text {adatom }}+\mu_{\text {total }} \mu_{s i}\right) \text {. }
$$

4 Band Structure

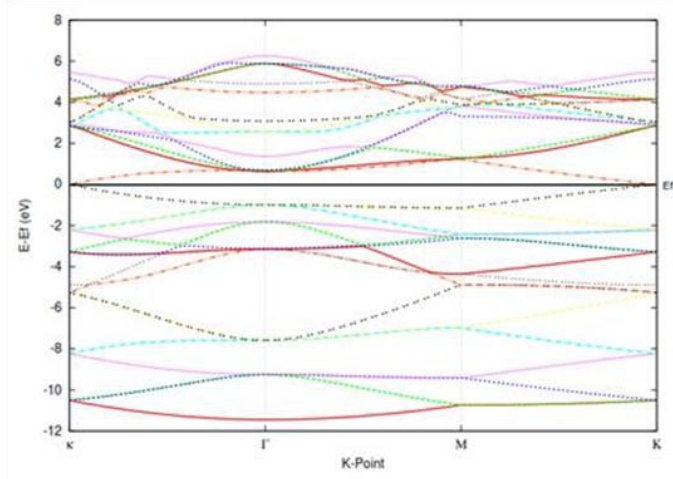

Figure 2. Band structure of pristine silicene with supercell $2 \times 2$.

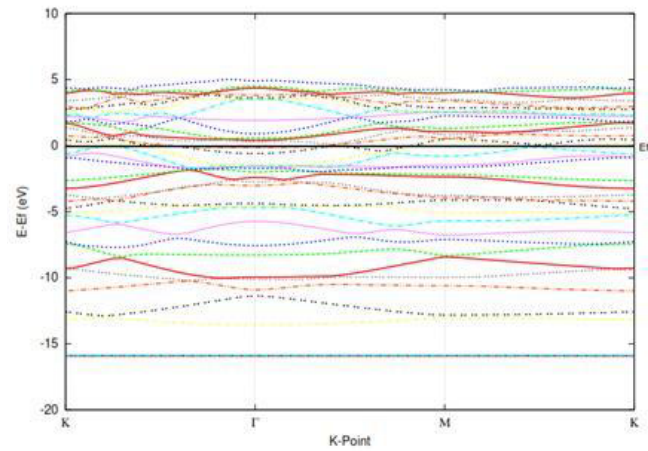

Figure 3. Band structure of silicene doped by $\mathrm{Ga}$ atom at B-site

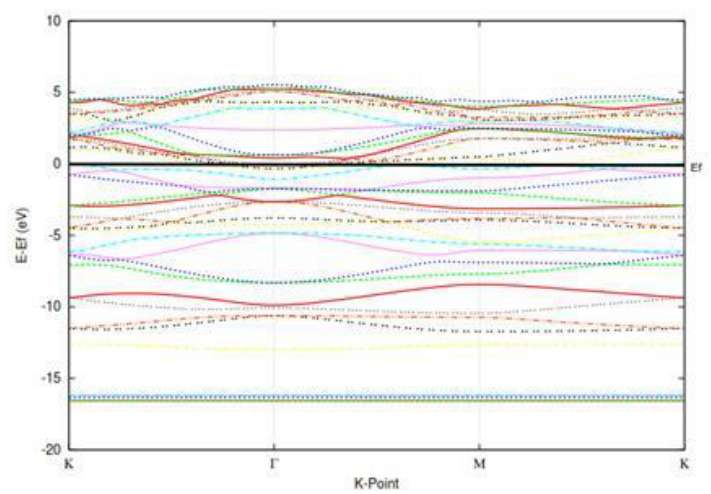

Figure 4. Band structure of silicene doped with $\mathrm{Ga}$ atom at H-Site.

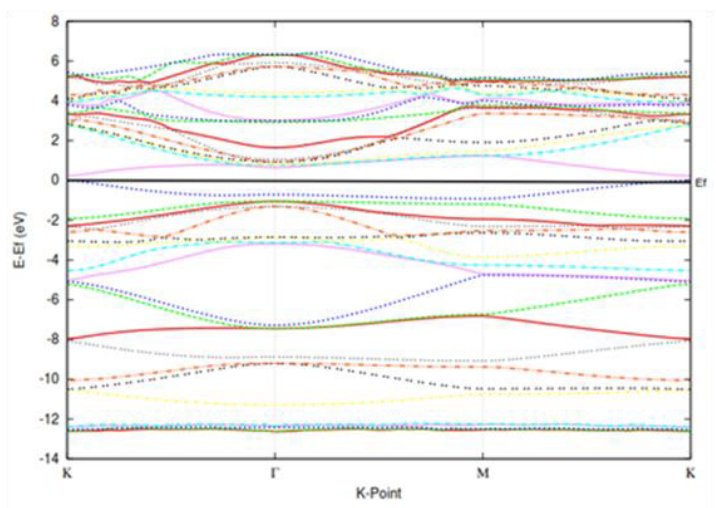

Figure 5. Band structure of silicene doped with $\mathrm{Ga}$ atom at $\mathrm{S}$ Site. 
Figure 2 shows that there is no gap between valence band and conduction band (zero band gap)at Fermi energy. There is also no overlap between valence band and conduction band. The two bands meet at $\mathrm{K}$ point.One can conclude thatpristine silicene is a semimetal.

After siliceneis doped withGaatom, its band structure changed. For Gaatom at H-Site, B-Site, and T site (Figure 3,4 and 5 respectively) there is no gap at Fermi energy, several energies level overlap. Therefore, there is no Dirac point. It can be inferred that silicene doped at those sites is conductor. While, silicene doped withGa atom at S-site(Figure 6)posses small gap at Fermi energy. It is a direct gap of $0,275 \mathrm{eV}$ which means silicene doped with $\mathrm{Ga}$ atom at $\mathrm{S}$-site is a semiconductor.

\section{Conclusion}

In summary, Ga doping on siliceneat B-site, T-site and $\mathrm{H}$-site changes it from semimetal to conductor. While at S-site, Ga atom changes silicene from semimetal to semiconductor with energy gap of $0,275 \mathrm{eV}$

\section{References}

1. A.H.C. Neto and K. Novoselov, REPORTS Prog. Phys. 74, (2011).

2. S. Fagan, R. Baierle, R. Mota, A. da Silva, and A. Fazzio, Phys. Rev. B 61, 9994 (2000).
3. P. Vogt, P. De Padova, C. Quaresima, J. Avila, E. Frantzeskakis, M.C. Asensio, A. Resta, B. Ealet, and G. Le Lay, Phys. Rev. Lett. 108, 155501 (2012).

4. L. Tao, E. Cinquanta, D. Chiappe, C. Grazianetti, M. Fanciulli, M. Dubey, A. Molle, and D. Akinwande, Nat. Nanotechnol. 10, 227 (2015).

5. N.D. Drummond, V. Zólyomi, and V.I. Fal'ko, Phys. Rev. B 85, 075423 (2012).

6. Z. Ni, Q. Liu, K. Tang, J. Zheng, J. Zhou, R. Qin, Z. Gao, D. Yu, and J. Lu, Nano Lett. 12, 113 (2012).

7. L. Chen, C.-C. Liu, B. Feng, X. He, P. Cheng, Z. Ding, S. Meng, Y. Yao, and K. Wu, Phys. Rev. Lett. 109, 056804 (2012).

8. C.-C. Liu, W. Feng, and Y. Yao, Phys. Rev. Lett. 107, 076802 (2011).

9. R Brako, D Šokčević, $\mathrm{P}$ Lazić and N Atodiresei, 2010 New J. Phys. 12 113016(2010).

10. S. Lebègue and O. Eriksson, Phys. Rev. B 79, 4 (2009).

11. B. Feng, Z. Ding, S. Meng, Y. Yao, X. He, P. Cheng, L. Chen, and K. Wu, Nano Lett. 12, 3507 (2012).

12. A.H.C. Neto, Rev. Mod. Phys. 81, 109 (2009). 